Marquette University

e-Publications@Marquette

Chemistry Faculty Research and Publications

Chemistry, Department of

$1-1-1994$

\title{
An m-Carboranedicarboxylic Acid Dianilide
}

Sergey V. Lindeman

Marquette University, sergey.lindeman@marquette.edu

Georgii A. Kats

Institute of Organoelement Compounds

Lyudmila G. Komarova

Institute of Organoelement Compounds

Published version. Acta Crystallographica Section C, Vol. 50 (1995): 1299-1301. DOI. (C) 1995

International Union of Crystallography. Used with permission. 
The support of the Herbert $\mathrm{H}$. and Grace A. Dow Foundation, The Dow Chemical Company Foundation and Central Michigan University in the establishment of the CMU X-ray facility is gratefully acknowledged.

Lists of structure factors, anisotropic displacement parameters and complete geometry have been deposited with the IUCr (Reference: CR 1091) Copies may be obtained through The Managing Editor, International Union of Crystallography, 5 Abbey Square, Chester CH1 2HU, England.

\section{References}

Beurskens, P. T., Bosman, W. P., Doesburg, H. M., Gould, R. O., van den Hark, Th. E. M., Prick, P. A., Noordik, J. H., Beurskens, G., Parthasarthi, V., Bruins Slot, H. J. \& Haltiwanger, R. C. (1983). DIRDIF. Technical Report 1983/1. Crystallography Laboratory, Toernooiveld, 6525 ED Nijmegen, The Netherlands.

Cromer, D. T. (1974). International Tables for X-ray Crystallography, Vol. IV, Table 2.3.1. Birmingham: Kynoch Press. (Present distributors Kluwer Academic Publishers, Dordrecht.)

Cromer, D. T. \& Waber, J. T. (1974). International Tables for $X$-ray Crystallography, Vol. IV, Table 2.2A. Birmingham: Kynoch Press. (Present distributors Kluwer Academic Publishers, Dordrecht.)

Gilmore, C. J. (1983). MITHRIL. Computer Program for the Automatic Solution of Crystal Structures from X-ray Data. Department of Chemistry, Univ. of Glasgow, Scotland.

Johnson, C. K. (1976). ORTEPII. Report ORNL-5138. Oak Ridge National Laboratory, Tennessee, USA.

Kosnic, E. J., McClymont, E. L., Hodder, R. A. \& Squattrito, P. J. (1992). Inorg. Chim. Acta, 201, 143-151.

Molecular Structure Corporation (1988). MSC/AFC Diffractometer Control Software. Revised edition. MSC, 3200 Research Forest Drive, The Woodlands, TX 77381, USA.

Molecular Structure Corporation (1991). TEXSAN. TEXRAY Structure Analysis Package. Revised edition. MSC, 3200 Research Forest Drive, The Woodlands, TX 77381, USA.

Zachariasen, W. H. (1968). Acta Cryst. A24, 212-216.

Acta Cryst. (1994). C50, 1299-1301

\section{An $m$-Carboranedicarboxylic Acid Dianilide}

\author{
Sergey V. Lindeman, Georgit A. Kats \\ AND LYUDMILA G. KOMAROVA
}

Institute of Organoelement Compounds, 28 Vavilov St., Moscow 117813, Russia

(Received 23 November 1993; accepted 19 January 1994)

\author{
Abstract \\ The crystal structure of the 'non hydrogen-bonded' \\ (according to IR data) polymorph of 1,7-bis(phenyl- \\ carbamoyl)-1,7-dicarba-closo-dodecaborane(12), $\quad \mathrm{C}_{16^{-}}$

$\mathrm{H}_{22} \mathrm{~B}_{10} \mathrm{~N}_{2} \mathrm{O}_{2}$, has been determined. The two phenylamide groups have a $Z$ configuration [the torsion angles $\mathrm{O}-\mathrm{C}-\mathrm{N}-\mathrm{C}$ are $-2.3(5)$ and $\left.-3.0(5)^{\circ}\right]$. As a result both 'active' protons of these groups are almost completely shielded by other $\mathrm{H}$ atoms of the neighbouring carborane nucleus and phenyl substituents, and, therefore, no hydrogen-bonding contacts are found.

\section{Comment}

It is widely known that the specific properties of polyamides depend strongly on the presence of interchain hydrogen-bonding interactions. The hydrogenbonding parameters may often be predicted on the basis of the crystal structures of low molecular weight analogues of these polymers. Such studies have been carried out, in particular, for low molecular weight models of the elementary units of aliphatic (Ruede \& Fayos, 1982) and aromatic (Brisson \& Brisse, 1985, 1986) nylons.

The title compound (I) was synthesized as a model of the elementary unit of poly $\left(4,4^{\prime}\right.$-diphenylene-1,7carboranyl dicarbamide), $\left[-\mathrm{OCCB}_{10} \mathrm{H}_{10} \mathrm{CCOHNC}_{6} \mathrm{H}_{4}\right.$ $\left.\mathrm{C}_{6} \mathrm{H}_{4} \mathrm{NH}-\right]_{n}$. It has been shown by IR specroscopy that the title compound (I) has two polymorphs (Leites, Kats, Bukalov \& Komarova, 1991). By using a slow crystallization method the stable 'non hydrogen-bonded' modification ( $\mathrm{I} a$ ) is formed, whereas fast crystallization leads to a metastable 'hydrogen-bonded' form ( $\mathrm{I} b$ ) in which $\mathrm{N}-\mathrm{H} \cdots \mathrm{O}$ bonds are present. The relative amount of each form obtained depends on the particular conditions used for precipitation. Unfortunately, we were only able to prepare suitable crystals of the (I $a$ ) form for this study.

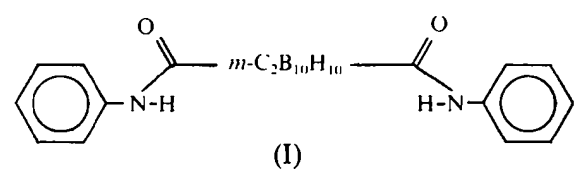

The molecule of (I) in form (Ia) is elongated in shape (Fig. 1). The geometrical parameters of this molecule are in good agreement with the standard values (Allen, Kennard, Watson, Brammer, Orpen \& Taylor, 1987). Both chemically equivalent halves of the molecule have a similar conformation: the carbonyl groups are synoriented with respect to the B1 and B2 atoms and the amide groups have a $Z$ configuration and are essentially planar. The terminal phenyl groups are rotated out of the planes of the adjacent amide groups [the corresponding dihedral angles are $46.9(7)^{\circ}$ for the C11-C16 phenyl group and $26.2(4)^{\circ}$ for the $\mathrm{C} 21-\mathrm{C} 26$ phenyl group]. In this conformation both of the amide $\mathrm{H}$ atoms happen to be almost completely shielded by other $\mathrm{H}$ atoms of the neighbouring carborane nucleus and phenyl groups (Fig. 2). Evidently, this is the main reason for the absence of hydrogen bonds in the structure of $(\mathrm{I} a)$. 




Fig. 1. Perspective view of the structure of the title compound showing the atomic labelling.

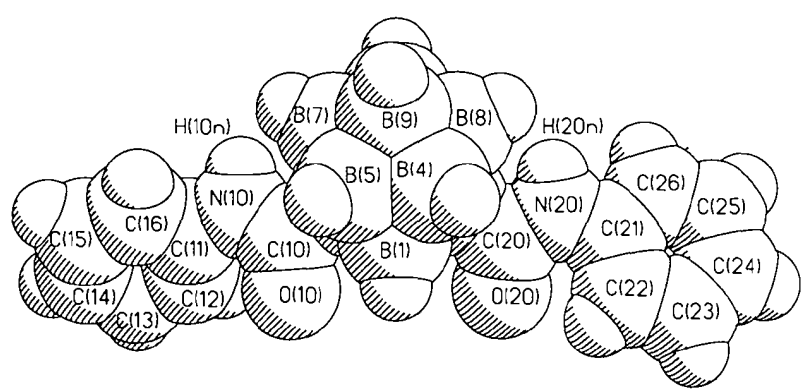

Fig. 2. Space-filling projection of the title compound indicating the spatial shielding of the amide $\mathrm{H}$ atoms.

In the case of the $E$ configuration of the amide groups, the $\mathrm{H}$ atoms of these groups would be exo-oriented and therefore more accessible to a hydrogen-bond acceptor. However, such a configuration would be much more strained due to the steric repulsion between the synoriented carborane nucleus and phenyl groups. These steric hindrances may be the cause of the metastability of the 'hydrogen-bonded' form ( $\mathrm{I} b)$, assuming that the structure of the latter is indeed built up from molecules of (I) in the $E$ configuration.

\section{Experimental \\ Crystal data}

$\mathrm{C}_{16} \mathrm{H}_{22} \mathrm{~B}_{10} \mathrm{~N}_{2} \mathrm{O}_{2}$

$M_{r}=382.46$

Triclinic

$P \overline{1}$

$$
\begin{aligned}
& a=9.727(3) \AA \\
& b=10.366(4) \AA \\
& c=10.727(3) \AA \\
& \alpha=106.36(3)^{\circ} \\
& \beta=96.08(3)^{\circ} \\
& \gamma=95.87(3)^{\circ} \\
& V=1022.0(6) \AA^{3} \\
& Z=2 \\
& D_{x}=1.243 \mathrm{Mg} \mathrm{m}^{-3}
\end{aligned}
$$

Mo $K \alpha$ radiation

$\lambda=0.71069 \AA$

Cell parameters from 24 reflections

$\theta=12-12.5^{\circ}$

$\mu=0.072 \mathrm{~mm}^{-1}$

$T=293(2) \mathrm{K}$

Well formed prisms

$0.4 \times 0.3 \times 0.25 \mathrm{~mm}$

Colourless

Data collection

Syntex $P 2_{1}$ diffractometer $\theta / 2 \theta$ scans

Absorption correction:

none
3775 measured reflections 3772 independent reflections 3009 observed reflections

$$
[I>2 \sigma(I)]
$$

$R_{\text {int }}=0.1131$

\section{Refinement}

Refinement on $F^{2}$

$R\left[F^{2}>2 \sigma\left(F^{2}\right)\right]=0.0505$

$w R\left(F^{2}\right)=0.1296$

$S=1.077$

3758 reflections

359 parameters

All $\mathrm{H}$-atom parameters refined

Calculated weights

$$
\begin{aligned}
& \begin{array}{l}
w= \\
\quad 1 /\left[\sigma^{2}\left(F_{o}^{2}\right)\right. \\
\quad+(0.0841 P)^{2} \\
\quad+0.1539 P]
\end{array} \\
& \text { where } P=\left(F_{o}^{2}+2 F_{c}^{2}\right) / 3
\end{aligned}
$$

2 standard reflections monitored every 98 reflections intensity variation: $2.5 \%$

$$
\begin{aligned}
& (\Delta / \sigma)_{\max }=0.018 \\
& \Delta \rho_{\max }=0.257 \mathrm{e}^{-3} \\
& \Delta \rho_{\min }=-0.180 \mathrm{e}^{-3} \\
& \text { Extinction correction: none } \\
& \text { Atomic scattering factors } \\
& \quad \text { from International Tables } \\
& \text { for Crystallography (1992, } \\
& \text { Vol. C, Tables } 4.2 .6 .8 \text { and } \\
& \text { 6.1.1.4) }
\end{aligned}
$$

Table 1. Fractional atomic coordinates and equivalent isotropic displacement parameters $\left(\AA^{2}\right)$

\begin{tabular}{lcccc}
\multicolumn{5}{c}{ isotropic displacement parameters $\left(\AA^{2}\right)$} \\
\multicolumn{5}{c}{$U_{\text {eq }}=(1 / 3) \sum_{i} \Sigma_{j} U_{i j} a_{i}^{*} a_{j}^{*} \mathbf{a}_{i} \cdot \mathbf{a}_{j}}$. \\
C1 & $x$ & $y$ & $z$ & $U_{\text {eq }}$ \\
C10 & $0.2504(2)$ & $0.8640(2)$ & $0.62916(15)$ & $0.0345(4)$ \\
O10 & $0.2887(2)$ & $0.9598(2)$ & $0.7688(2)$ & $0.0422(4)$ \\
N10 & $0.4087(2)$ & $1.0038(2)$ & $0.81282(15)$ & $0.0799(6)$ \\
C11 & $0.1807(2)$ & $0.9875(2)$ & $0.8334(2)$ & $0.0530(4)$ \\
C12 & $0.1899(2)$ & $1.0698(2)$ & $0.9667(2)$ & $0.0433(4)$ \\
C13 & $0.2838(2)$ & $1.0530(2)$ & $1.0639(2)$ & $0.0529(5)$ \\
C14 & $0.2833(3)$ & $1.1295(2)$ & $1.1931(2)$ & $0.0588(6)$ \\
C15 & $0.1916(3)$ & $1.2216(2)$ & $1.2229(2)$ & $0.0592(6)$ \\
C16 & $0.1012(3)$ & $1.2401(2)$ & $1.1256(2)$ & $0.0669(6)$ \\
C2 & $0.0988(3)$ & $1.1633(2)$ & $0.9962(2)$ & $0.0594(6)$ \\
C20 & $0.3471(2)$ & $0.7028(2)$ & $0.43101(15)$ & $0.0360(4)$ \\
O20 & $0.4791(2)$ & $0.6483(2)$ & $0.3831(2)$ & $0.0447(4)$ \\
N20 & $0.5901(2)$ & $0.6855(2)$ & $0.4519(2)$ & $0.0911(7)$ \\
C21 & $0.4583(2)$ & $0.5612(2)$ & $0.2625(2)$ & $0.0504(4)$ \\
C22 & $0.5594(2)$ & $0.4998(2)$ & $0.1885(2)$ & $0.0437(4)$ \\
C23 & $0.6964(2)$ & $0.5560(3)$ & $0.2076(2)$ & $0.0572(5)$ \\
C24 & $0.7868(3)$ & $0.4907(3)$ & $0.1270(2)$ & $0.0681(7)$ \\
C25 & $0.7400(3)$ & $0.3705(3)$ & $0.0302(2)$ & $0.0671(7)$ \\
C26 & $0.6027(3)$ & $0.3153(2)$ & $0.0112(2)$ & $0.0606(6)$ \\
B1 & $0.5115(3)$ & $0.3788(2)$ & $0.0901(2)$ & $0.0510(5)$ \\
B2 & $0.3762(2)$ & $0.8657(2)$ & $0.5309(2)$ & $0.0370(4)$ \\
B3 & $0.3357(2)$ & $0.7252(2)$ & $0.5928(2)$ & $0.0356(4)$ \\
B4 & $0.1551(2)$ & $0.7069(2)$ & $0.6030(2)$ & $0.0404(4)$ \\
B5 & $0.2850(2)$ & $0.8261(2)$ & $0.3703(2)$ & $0.0427(5)$ \\
B6 & $0.2207(2)$ & $0.9339(2)$ & $0.5030(2)$ & $0.0435(5)$ \\
B7 & $0.2203(2)$ & $0.5992(2)$ & $0.4709(2)$ & $0.0408(4)$ \\
B8 & $0.0837(2)$ & $0.8349(2)$ & $0.5470(2)$ & $0.0416(5)$ \\
B9 & $0.1873(2)$ & $0.6617(2)$ & $0.3339(2)$ & $0.0417(5)$ \\
B10 & $0.1032(2)$ & $0.8068(2)$ & $0.3802(2)$ & $0.0456(5)$ \\
& $0.0627(2)$ & $0.6669(2)$ & $0.4427(2)$ & $0.0432(5)$
\end{tabular}

Table 2. Selected geometric parameters $\left(\AA \mathrm{,}^{\circ}\right)$

$\begin{array}{llll}\mathrm{C} 1-\mathrm{C} 10 & 1.529(2) & \mathrm{C} 2-\mathrm{C} 20 & 1.535(2) \\ \mathrm{C} 1-\mathrm{B} 1 & 1.700(3) & \mathrm{C} 2-\mathrm{B} 2 & 1.702(2) \\ \mathrm{C} 1-\mathrm{B} 2 & 1.705(3) & \mathrm{C} 2-\mathrm{B} 1 & 1.702(3) \\ \mathrm{C} 1-\mathrm{B} 7 & 1.716(3) & \mathrm{C} 2-\mathrm{B} 8 & 1.719(3) \\ \mathrm{C} 1-\mathrm{B} 5 & 1.718(3) & \mathrm{C} 2-\mathrm{B} 6 & 1.720(3) \\ \mathrm{C} 1-\mathrm{B} 3 & 1.723(3) & \mathrm{C} 2-\mathrm{B} 4 & 1.722(3) \\ \mathrm{C} 10-\mathrm{O} 10 & 1.203(2) & \mathrm{C} 20-\mathrm{O} 20 & 1.202(2) \\ \mathrm{C} 10-\mathrm{N} 10 & 1.331(2) & \mathrm{C} 20-\mathrm{N} 20 & 1.334(2) \\ \mathrm{N} 10-\mathrm{C} 11 & 1.432(2) & \mathrm{N} 20-\mathrm{C} 21 & 1.419(2) \\ \mathrm{C} 11-\mathrm{C} 12 & 1.373(3) & \mathrm{C} 21-\mathrm{C} 22 & 1.369(3) \\ \mathrm{C} 11-\mathrm{C} 16 & 1.374(3) & \mathrm{C} 21-\mathrm{C} 26 & 1.391(3)\end{array}$




$\begin{array}{lllr}\mathrm{C} 10-\mathrm{C} 1-\mathrm{B} 1 & 114.93(14) & \mathrm{C} 20-\mathrm{C} 2-\mathrm{B} 2 & 115.73(14) \\ \mathrm{C} 10-\mathrm{C} 1-\mathrm{B} 2 & 115.81(14) & \mathrm{C} 20-\mathrm{C} 2-\mathrm{B} 1 & 113.97(14) \\ \mathrm{C} 10-\mathrm{C} 1-\mathrm{B} 7 & 121.77(14) & \mathrm{C} 20-\mathrm{C} 2-\mathrm{B} 8 & 122.67(14) \\ \mathrm{C} 10-\mathrm{C} 1-\mathrm{B} 5 & 118.18(14) & \mathrm{C} 20-\mathrm{C} 2-\mathrm{B} 6 & 120.46(15) \\ \mathrm{C} 10-\mathrm{C} 1-\mathrm{B} 3 & 119.68(14) & \mathrm{C} 20-\mathrm{C} 2-\mathrm{B} 4 & 117.71(14) \\ \mathrm{O} 10-\mathrm{C} 10-\mathrm{N} 10 & 124.8(2) & \mathrm{O} 20-\mathrm{C} 20-\mathrm{N} 20 & 125.1(2) \\ \mathrm{O} 10-\mathrm{C} 10-\mathrm{C} 1 & 120.4(2) & \mathrm{O} 20-\mathrm{C} 20-\mathrm{C} 2 & 120.4(2) \\ \mathrm{N} 10-\mathrm{C} 10-\mathrm{C} 1 & 114.8(2) & \mathrm{N} 20-\mathrm{C} 20-\mathrm{C} 2 & 114.5(2) \\ \mathrm{C} 10-\mathrm{N} 10-\mathrm{C} 11 & 125.3(2) & \mathrm{C} 20-\mathrm{N} 20-\mathrm{C} 21 & 128.0(2) \\ \mathrm{C} 12-\mathrm{C} 11-\mathrm{C} 16 & 120.5(2) & \mathrm{C} 22-\mathrm{C} 21-\mathrm{C} 26 & 120.5(2) \\ \mathrm{C} 12-\mathrm{C} 11-\mathrm{N} 10 & 121.1(2) & \mathrm{C} 22-\mathrm{C} 21-\mathrm{N} 20 & 123.2(2) \\ \mathrm{C} 16-\mathrm{C} 11-\mathrm{N} 10 & 118.3(2) & \mathrm{C} 26-\mathrm{C} 21-\mathrm{N} 20 & 116.4(2) \\ \mathrm{B} 1-\mathrm{C} 1-\mathrm{C} 10-\mathrm{O} 10 & -18.3(4) & \mathrm{B} 1-\mathrm{C} 2-\mathrm{C} 20-\mathrm{O} 20 & 32.6(4) \\ \mathrm{B} 2-\mathrm{C} 1-\mathrm{C} 10-\mathrm{O} 10 & 52.8(4) & \mathrm{B} 2-\mathrm{C} 2-\mathrm{C} 20-\mathrm{O} 20 & -38.1(4) \\ \mathrm{C} 11-\mathrm{N} 10-\mathrm{C} 10-\mathrm{O} 10 & -2.3(5) & \mathrm{C} 21-\mathrm{N} 20-\mathrm{C} 20-\mathrm{O} 20 & -3.0(5)\end{array}$

Program used to solve structure: SHELXTL-Plus (Sheldrick, 1989). Program used to refine structure: SHELXL93 (Sheldrick, 1993).

Lists of structure factors, anisotropic displacement parameters, $\mathrm{H}$-atom coordinates and complete geometry have been deposited with the $\mathrm{IUCr}$ (Reference: AB1141). Copies may be obtained through The Managing Editor, International Union of Crystallography, 5 Abbey Square, Chester $\mathrm{CH} 1$ 2HU, England.

\section{References}

Allen, F. H., Kennard, O., Watson, D. G., Brammer, L., Orpen, A. G. \& Taylor, R. (1987). J. Chem. Soc. Perkin Trans. 2, pp. S1-S19.

Brisson, J. \& Brisse, F. (1985). Can. J. Chem. 63 3390-3397.

Brisson, J. \& Brisse, F. (1986). Macromolecules, 19, 2632-2639.

Leites, L. A., Kats, G. A., Bukalov S. S. \& Komarova, L. G. (1991). Izv. Akad. Nauk SSSR Ser. Khim. pp. 371-376.

Ruede, D. R. \& Fayos, J. (1982). Eur. Polym. J. 18, 1-4.

Sheldrick, G. M. (1989). SHELXTL-Plus. PC version. Siemens Analytical X-ray Instruments Inc., Madison, Wisconsin, USA.

Sheldrick, G. M. (1993). SHELXL93. Program for the Refinement of Crystal Structures. Univ. of Göttingen, Germany. 